\title{
Polysarcosine-Functionalized Lipid Nanoparticles for Therapeutic mRNA Delivery
}

Sara S. Nogueira, Anne Schlegel, Konrad Maxeiner, Benjamin Weber, Matthias Barz, Martin A. Schroer, Clement E. Blanchet, Dmitri I. Svergun, Srinivas Ramishetti, Dan Peer, Peter Langguth, Ugur Sahin, and Heinrich Haas*

Cite This: ACS Appl. Nano Mater. 2020, 3, 10634-10645

Read Online

ACCESS | 네 Metrics \& More | 回 Article Recommendations | sl Supporting Information

ABSTRACT: Polysarcosine (pSar) is a polypeptoid based on the endogenous amino acid sarcosine (N-methylated glycine), which has previously shown potent stealth properties. Here, lipid nanoparticles (LNPs) for therapeutic application of messenger RNA were assembled using pSarcosinylated lipids as a tool for particle engineering. Using pSar lipids with different polymeric chain lengths and molar fractions enabled the control of the physicochemical characteristics of the LNPs, such as particle size, morphology, and internal structure. In combination with a suited ionizable lipid, LNPs were assembled, which displayed high RNA transfection potency with an improved safety profile after intravenous

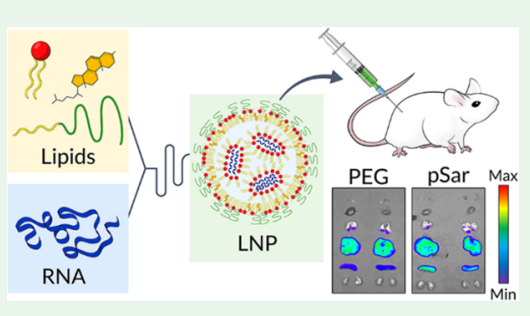
injection. Notably, a higher protein secretion with a reduced immunostimulatory response was observed when compared to systems based on polyethylene glycol (PEG) lipids. pSarcosinylated nanocarriers showed a lower proinflammatory cytokine secretion and reduced complement activation compared to PEGylated LNPs. In summary, the described pSar-based LNPs enable safe and potent delivery of mRNA, thus signifying an excellent basis for the development of PEG-free RNA therapeutics.

KEYWORDS: lipid nanoparticles, LNPs, mRNA, drug delivery, gene delivery, precision medicine, polysarcosine, SAXS

$\mathrm{M}$ essenger RNA (mRNA) delivery holds great promise for various therapeutic applications, including protein replacements, immunotherapy, and genome editing. ${ }^{1}$ The mRNA platform offers several benefits over other nucleic acidbased therapeutics as mRNA is non-infectious, it does not require transport to the nucleus and it does not integrate into the host genome. mRNA is generally subject to rapid degradation in biological fluids, which can be considered useful from a safety perspective. However, for application of the mRNA as a pharmaceutical product, chemical modification or encapsulation into carrier vehicles is required for delivery to the target compartment while maintaining sufficient in vivo stability. ${ }^{1,2}$

Lipid-based carrier vehicles have shown a significant translational promise in the delivery of mRNA in clinical trials. $^{3-5}$ One approach for assembling lipid nanocarriers, initially developed for siRNA delivery, includes direct mixing of a solution of lipids in ethanol with an aqueous solution of the nucleic acid to obtain lipid nanoparticles (LNPs). ${ }^{6}$ Recently, siRNA-LNPs were approved by the FDA for the treatment of polyneuropathies induced by hereditary transthyretin amyloidosis (Onpattro). ${ }^{7}$ In general, LNPs comprise four primary components: (1) an ionizable-cationic lipid or lipidoid compound, which complexes the negatively charged RNA and improves endosomal escape through its $\mathrm{pH}$-dependent charge; (2) cholesterol, which enhances LNP stability and promotes membrane fusion; (3) a phospholipid that structuralizes the lipid bilayer and optionally facilitates endosomal escape through its fusogenic properties, and (4) polyethylene glycol (PEG)-conjugated lipid, which prevents aggregation during particle formation and allows the controlled manufacturing of particles with defined diameters in the range between approximately 50 and $150 \mathrm{~nm} .{ }^{8}$

In order to allow nanoparticle formation using an LNP platform, virtually all research groups have been using PEGconjugated lipids. PEG has also been extensively used as a stealth coating in the pharmaceutical industry. It provides a hydrophilic steric hindrance resulting in reduced protein opsonization, increased circulation times, and improved particle pharmacokinetics. However, the PEGylation of nanoparticles can also have substantial disadvantages concerning activity and safety, which is named the "PEG dilemma". PEGylation may lead to the lowering of the transfection potency by hindrance cellular uptake and processing. It has been associated with the accelerated blood clearance (ABC) phenomenon, typically induced by the formation of anti-PEG antibodies following repeated systemic administration. ${ }^{9-12}$ These anti-PEG antibodies are typically found within healthy

Received: July 8, 2020

Accepted: September 25, 2020

Published: September 25, 2020 
humans due to the increasingly probability of exposure to PEG-containing products, such as in cosmetics. ${ }^{12,13}$ PEG has been shown to trigger complement activation, which can lead to hypersensitivity reactions, also known as complement activation-related pseudo-allergy (CARPA) in specific individuals. ${ }^{14,15}$ The drawbacks associated with the therapeutic use of PEGylated nanoparticles has stimulated the development of PEG alternatives, such as poly(glycerol) (PGs), poly(oxazolines), sugar-based systems, and poly(peptide)s. ${ }^{16,17}$

In recent years, a class of polymers based on amino acid derivatives, the polypeptoids, has been explored as a substitute for PEG in attaining stealth properties in products for biomedical applications. ${ }^{18}$ Among those, polysarcosine (pSar), a polymer made of repetitive units of the endogenous amino acid sarcosine (N-methylated glycine), exhibited stealth-like properties comparable to PEG products. ${ }^{19-27}$ Maurer et al. reported on the low immunogenicity of pSar lipids in rabbits and rats, while Feneroli and co-workers observed an absence of an acute immune response in zebrafish embryos and mice. ${ }^{21,28}$ Several studies dealt with the preparation of sarcosine-based drug carriers. With the present study, this concept has been extended to the use of pSarcosinylated lipids for the assembly of lipid nanoparticles as RNA delivery systems.

In this work, we used pSar lipids as a tool for engineering mRNA-LNPs with improved biological activity and safety. The pSarcosinylated lipids were applied instead of using PEGylated lipids for size control within manufacturing of LNPs based on established rapid mixing protocols. ${ }^{29}$ The influence of the molar fraction and the number of pSar repeat units on the physicochemical characteristics of the resulting particle systems was thoroughly investigated and correlated with biological activity. Hydrophilic pSar chain length was varied whilst maintaining a consistent hydrophobic hydrocarbon moiety to optimize the balance between stealth requirements and facility of cellular uptake. pSar lipid technology was further validated by assembling pSar LNPs that comprised further ionizable cationic lipids with improved transfection efficacy. Intravenous administration of such pSar systems substantially boosted the mRNA expression in vivo. Finally, liver toxicity and potential immunostimulatory of pSar-based lipid nanoparticles were evaluated in comparison to established PEGylated lipid-based formulations.

\section{RESULTS AND DISCUSSION}

pSarcosinylated Lipids as a Tool for Particle Engineering. Initial experiments focused on the investigation of pSar lipids for engineering mRNA nanoparticles using the LNP rapid mixing protocol. ${ }^{29}$ The pSar lipids used in this work have been synthesized according to Weber et al. ${ }^{26,30}$ They contain a single aliphatic carbon chain and a secondary amine as an end group. pSarcosinylated lipids with different pSar chain lengths (average of 11-65 sarcosine repeat units) and at different molar fractions $(0-10 \%)$ were investigated for LNP manufacturing. In addition to the pSar lipids, the cationic ionizable lipid DODMA, the helper lipid DSPC, and cholesterol at respective molar fractions $40 / 10 /(50-x)$ were used, where $x$ is the fraction of the pSar lipid (Figure 1 and Supporting Table 1). Ethanolic solutions containing the lipids were mixed with an aqueous phase containing modified mRNA, at a nitrogen to phosphate $(\mathrm{N} / \mathrm{P})$ ratio of 4 , using a commercially available microfluidic device. ${ }^{29}$ For comparison, nanoparticles with the same lipid composition were assembled

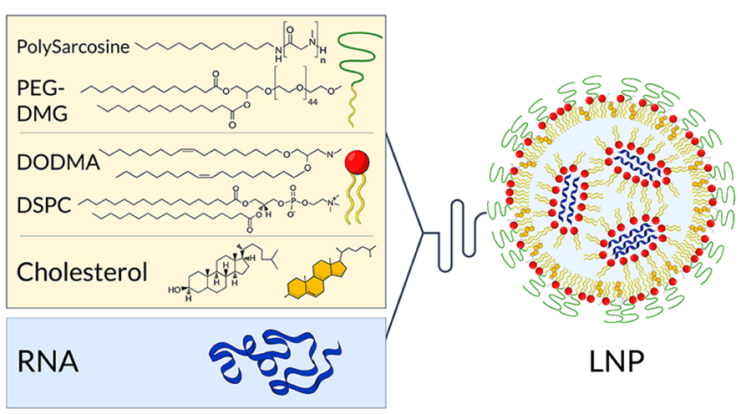

Figure 1. Lipids used for LNP manufacturing. LNPs are generally composed of ionizable amino lipids, helper lipids, cholesterol, and a stealth moiety. Here, as lipids, the ionizable 1,2-dioleyloxy-3dimethylaminopropane (DODMA) and the phospholipid 1,2distearoyl-sn-glycero-3-phosphocholine (DSPC) were used. As the stealthy moiety, either 1,2-dimyristoyl-rac-glycero-3-methylpolyoxyethylene (PEG-DMG) or pSar lipids were used. For pSar, $n$ represents the number of sarcosine repeating unit. The composition of the LNPs was $40 / 10 / 50-x / x$ (DODMA/DSPC/cholesterol/grafted lipid, molar ratio), where $x$ is the fraction of the grafted lipid. The cartoon at the right shows an artistic representation of the LNPs: the RNA is thought to be present embedded in the excess lipid matrix, in the form of organized stacks of few repeat units, complexed with lipid membranes

with PEG-DMG, a commercially available PEG lipid, instead of the pSar lipids.

Lipid particle formulations containing no grafted lipid aggregated immediately post-production (data not shown). In contrast, however, the incorporation of pSar lipid enabled the formation of colloidally stable lipid nanoparticles, where the size depended on the chain length and the molar fraction of pSar lipids (Figure 2A). For all pSar chain lengths, the particle sizes decreased with the increased amounts of pSar lipid in LNP compositions, similar to PEGylated systems (Figure 2B). ${ }^{29,31}$ The particle size decreased also with increasing pSar length. In absolute numbers, the particles comprising pSar were larger than those obtained with PEG lipids at a similar molar fraction. For instance, LNPs formed with $1.5 \mathrm{~mol}^{\%} \mathrm{pSar}_{34}$ (which has an equivalent molecular weight to PEG-DMG) exhibited a diameter of about $150 \mathrm{~nm}$, while with PEG-DMG, it was $90 \mathrm{~nm}$. All particles exhibited a PdI lower than 0.25 and a close to neutral $\zeta$-potential at $\mathrm{pH} 7.4$ (data not shown). Further differences between the PEG and the pSar LNPs could be observed for the accessibility of the particle-bound RNA to fluorescent dye molecules (Ribogreen assay, Figure 2C). Although, for the particles formed at this $\mathrm{N} / \mathrm{P}$ ratio and with this lipid composition, in general no substantial amounts of free RNA were determined (here exemplary demonstrated by agarose gel measurements for a pSar and a PEG system, Figure 2D) the RNA inside the pSar particles showed a systematically higher accessibility to the dye than that in the particles formed with PEG. This high accessibility points toward differences in the compactness inside the pSar and PEG nanoparticles, which may have consequences for the stability and activity. Lower packing compactness may be favorable for endosomal processing, while higher compactness may be favorable for stability with respect to enzymatic RNA degradation in circulation. However, the risk of elevated degradation is lower than it would be suggested by the dye accessibility because enzymes are much bigger than the dye molecules. Overall, the observed effects of both types 

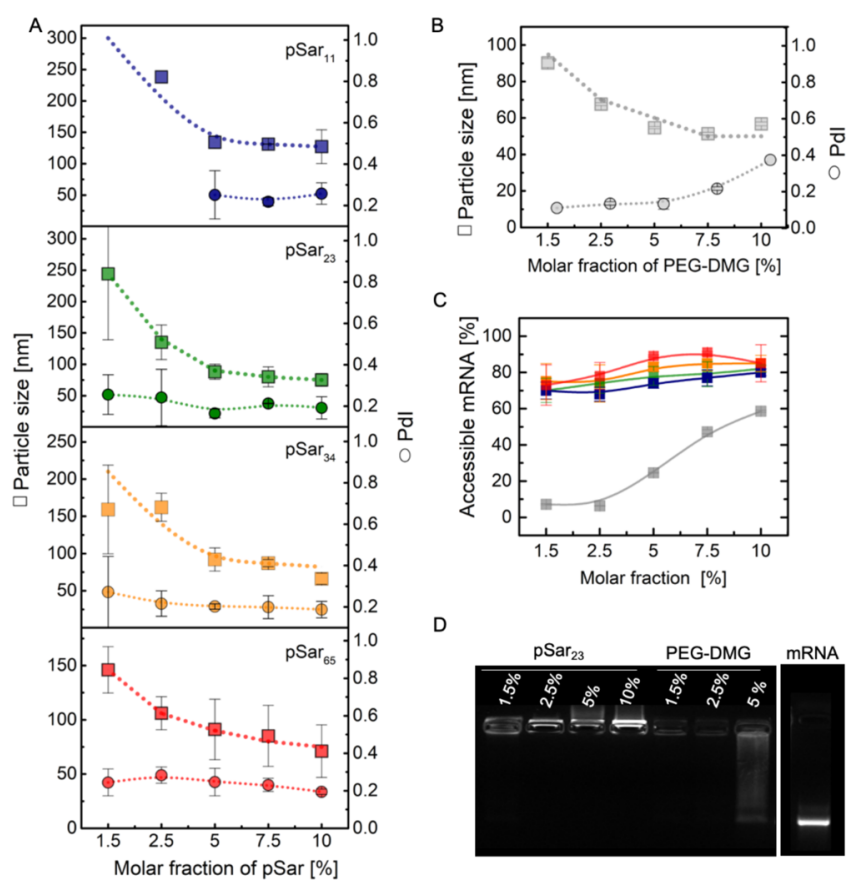

C

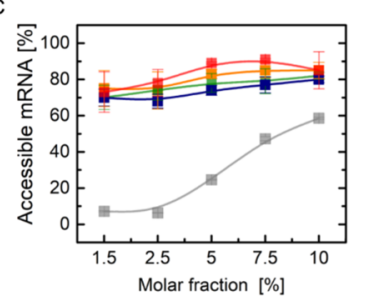

D

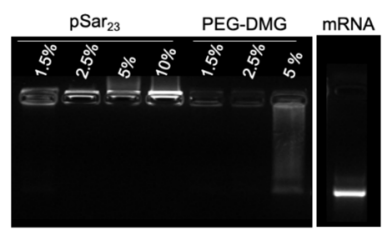

Figure 2. Effect of pSar lipid on LNP characteristics compared to PEG lipid. (A) Size (hydrodynamic diameter) and polydispersity index (PdI) as obtained from dynamic light scattering measurements of LNPs as a function of molar fraction (1.5, 2.5, 5, 7.5, and 10\%) of the pSar moieties with varying degrees of polymerization (blue squares, 11; green squares, 23; orange squares, 34 ; red squares, 65 ). Data are presented as mean \pm standard deviation for $n=2-3$. (B) Size and PdI of LNPs as a function of molar fraction of PEG-DMG (gray squares, PEG-DMG). (C) Accessibility of the RNA to dye molecules using the Ribogreen kit. Systematically higher accessibility is measured for the pSar LNPs than for those comprising PEG. (D) Determination of free RNA by the agarose gel assay. Both LNPs with PEG and pSar show equally low values for free RNA. All LNPs had the same basic composition, with the molar ratios DODMA//DOPE/ cholesterol/grafted lipid 40/10/50 - x/ $x$. In all cases, the N/P ratio (DODMA to RNA), calculated as outlined in the Methods section, was 4 . (Data presented as mean \pm standard deviation, $n=1$.)

of grafted lipids on LNP design demonstrate their applicability for particle engineering but require individual adjustment.

The internal organization of the pSarcosinylated and PEGylated LNPs was investigated by small-angle X-ray scattering (SAXS) (Figure 3 and Supporting Figure 1). The scattering profiles display similar features, in which a single broad maximum dominates the curves at the momentum transfer, $q$, of about $1 \mathrm{~nm}^{-1}$, corresponding to the spacing of about 6-7 $\mathrm{nm}$ from the application of Bragg's law (Methods section, eq 5). In all cases, the peaks became less pronounced increasing fractions of grafted lipid. Interestingly, some curves display a further modulation of intensity at a very low $q$, with a maximum at about $0.01 \mathrm{~nm}^{-1}$, which is most clearly visible for the data from the PEG systems (highlighted in Supporting Figure 2). Such a pattern could result from the overall particle form factor (see the Methods section, eqs 2 and 3). With a model of a solid sphere, one would expect oscillations with the first minimum at $q \times R=4.49$. Estimating the minimum position before the maximum to $q=0.05-0.09 \mathrm{~nm}^{-1}$, one obtains $R=90-50 \mathrm{~nm}$, which is roughly in the order of the DLS results (taking also into account that anisotropy and surface roughness of the particles lead to deviations from the solid sphere model). The curves are otherwise smooth and exhibit a steep intensity decay as a function of $q$, where a region of linear slope in the $q$ range below the main peak can be identified.

Quantitative data analysis underlines these qualitative observations. By using Lorentzian functions, the peak positions, peak width, and peak area were determined, from which information on repeat distance, correlation length, and relative amount of material present in such organization can be derived (see the Methods section for details), as shown in Figure 3C (Supporting Information shown in Supporting Figure 1 and Supporting Table 2). A slight shift of peak positions (top diagram) as a function of the molar fraction of the grafted lipid, corresponding to an increase of the repeat distance, is discernible. This could be due to partial insertion of the bulky stealthy lipids into the ordered stacks. The peak width, indicating the correlation length, ${ }^{34} \xi$, (see the Methods section, eq 6), increased slightly, and therefore the correlation length slightly decreased.

As was already visible by the eye, the peak area decreased with increasing fraction and chain length of the grafted lipid, with most pronounced effects observed for the PEG-DMG data (Figure 3 and Supporting Figure 1).

The above hypothesized partial insertion of the grafted lipids into the bilayer stacks may be a plausible explanation for the findings. Their large hydrophilic moieties can account for the decrease of order as well as for the shift of the peak positions toward a lower $q$ (larger spacing). Different chemical structures of the PEGylated and pSar lipids moiety may be the reason for the larger effect of the PEGylated LNPs.

These peak characteristics are in agreement with the presence of particles having a low internal organization, in the sense that some organized stacks are present, but only with few repeat units. Data sets from samples where no more peaks are discernible are indicative of the absence of ordered structures. Accordingly, calculation of the correlation length from the peak widths using a model for liquid crystals results in correlation lengths in the same order of magnitude as the repeat distance itself (see Methods and Supporting Information sections). This conjecture is further underlined by comparison of the experimental data with simulations using very basic assumptions for form factor and structure factor (see Methods and Supporting Information sections). The scattering curves can be fairly well represented by organizing only two to three repeat units at the distances indicated by the peak position (eqs 2, 7, and 8 in the Methods section and Supporting Figure 2).

The range of smooth decay at lower $q$ values was analyzed using a power law $\left(I \approx q^{-x}\right)$, where from the exponent, $x$, the mass fractal dimension of the particles can be derived (Porod exponent, eq 4). ${ }^{32,33}$ A Porod exponent of -4 is indicative of ideally smooth interfaces, lower (negative) numbers, e.g., -4 to -3 , is thought to be induced by surface roughness (surface fractals), while slopes between -3.5 and -2 point toward mass fractals. Here, a decrease of the Porod exponents (Figure 3 and Supporting Figure 1) with increasing fractions of grafted lipid was determined. Therefore, the surface and/or the mass fractal dimension increased. Molecularly spoken, higher surface roughness, changes in packing, or a higher anisotropy could account for such a change in slope. It may be worthwhile mentioning that the Porod exponents observed here for LNPs were still relatively high in comparison to other types of RNA nanoparticle (hybrid polymer and lipid) systems, where values up to -2 were determined. ${ }^{35}$ 
A

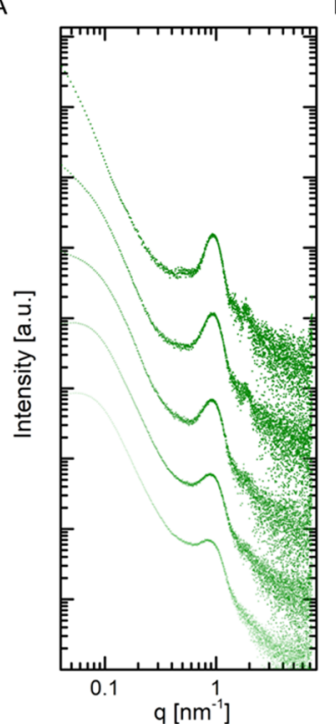

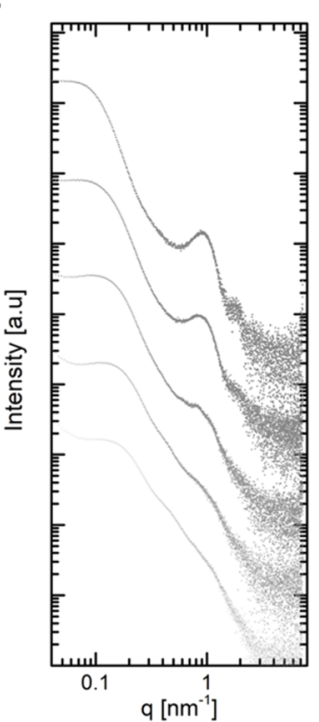

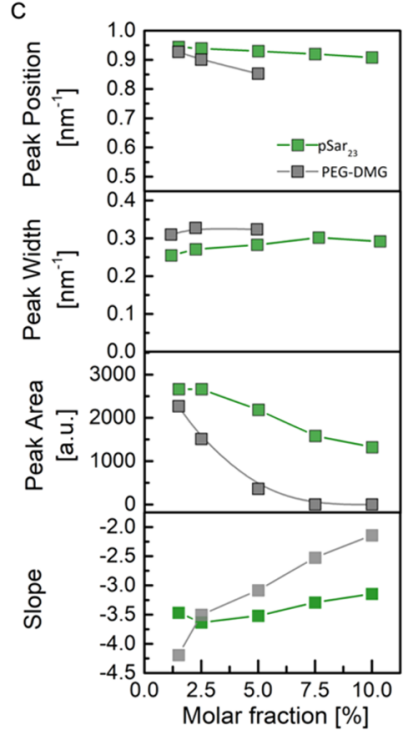

Figure 3. Small angle X-ray scattering (SAXS) data from $\mathrm{pSar}_{23}$ and PEG formulations. (A, B) SAXS curves from pSar ${ }_{23}$ and PEG-DMG-based LNPs for different molar fractions of the grafted lipids (1.5, 2.5, 5, 7.5, and $10 \mathrm{~mol} \%$ from top to bottom). In (C), peak position, peak width, and peak area as a function of molar fraction of $\mathrm{pSar}_{23}$ and PEG-DMG are presented in the first three diagrams from top to bottom. For the PEG-DMG systems, the peak area at the higher fractions was too low to be clearly quantified; therefore, no values for area and position are given. The fourth diagram shows the Porod exponent for the scattering curves, determined as described in the text. Further information can be found within the Methods and Supporting Information sections.
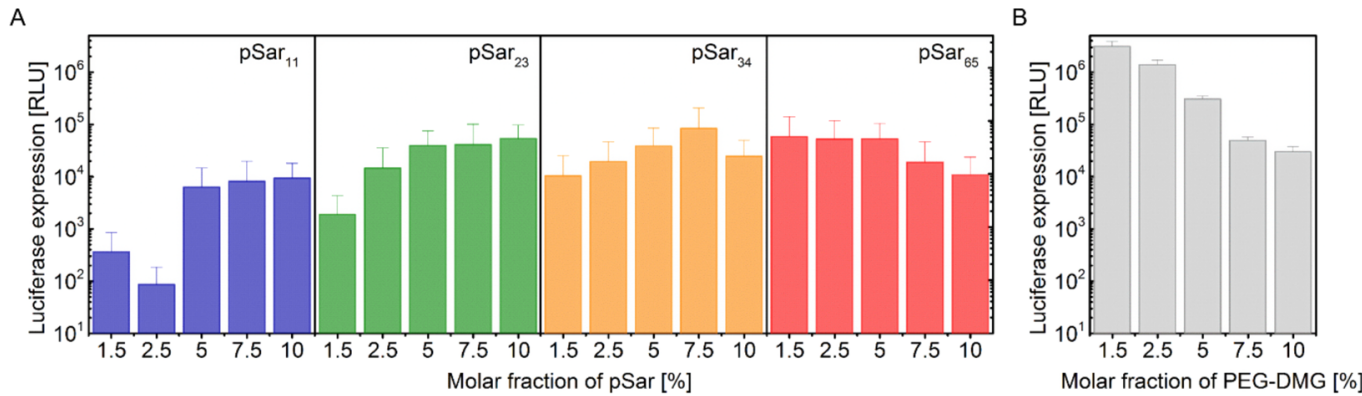

Figure 4. In vitro expression profile of $\mathrm{pSar}_{x}$ and PEG LNPs in a human hepatocyte cell line (HepG2). Firefly luciferase expression $24 \mathrm{~h}$ postincubation with $250 \mathrm{ng} / \mathrm{mL}$ mRNA-loaded LNPs comprising increased molar fractions of (A) pSar ${ }_{x}$ and (B) PEG-DMG. Data are represented as mean \pm standard deviation, $n=3$.

The data presented here allow one to further refine the understanding of the molecular organization inside LNPs as obtained in earlier studies. Although, so far, only spare systematic data from X-ray scattering measurements have been reported, ${ }^{38,39}$ a variety of investigations with various independent methods, ranging from cryo-TEM measurements to molecular modeling, has been performed. Based on these studies, the LNPs were frequently described as compact "electron-dense" particles with the RNA embedded in an excess of unorganized lipid matrix. The RNA is often assumed to be present either in a hexagonal organization or in the form of individual inverse micelle (or "current bun") organization. $^{36,37}$ The quantitative analysis of the present SAXS measurements points toward an internal organization in between the two above-described options. With this low organization, for the present samples, it is not meaningful to derive a specific type of order, e.g., hexagonal, lamellar, or cubic. The degree of organization further decreased with increasing fractions of grafted lipids. The cases, where no more peaks were visible, would be in accordance with the presence of individual lipoplexed RNA micelles (the individual currents in the bun), which are not correlated to each other. This conjecture is further highlighted by comparison of the experimental data with simulations using basic formalisms (see Methods and Supporting Information sections), which demonstrates that already two to three organized scattering units allow to reproduce characteristic aspects of the experimental scattering curves fairly well.

Of note is the general observation that the structure of LNPs as measured here differs substantially from so-called lipoplex systems, which may comprise similar lipids, but where lipids in an aqueous phase (liposomes) instead of the ethanolic lipid solution are mixed with the RNA. These have been investigated much more thoroughly than it has been done so far for the LNP systems. ${ }^{40-45}$ Studies with a wide variety of lipid compositions in combination different types of nucleotides (i.e., DNA, siRNA, mRNA) have been performed, resulting in a profound understanding of structural coherencies and internal phase properties. For these lipoplexes, typically much narrower Bragg peaks up to several orders can be observed, often pointing toward lamellar organization. ${ }^{46,47}$ LNPs appear to be characterized a much lower internal organization in comparison to lipoplexes. Taking into account that LNPs and lipoplexes have proven to be preferential for 


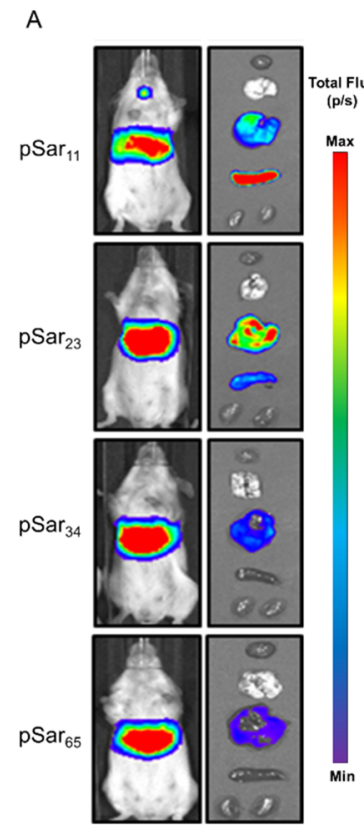

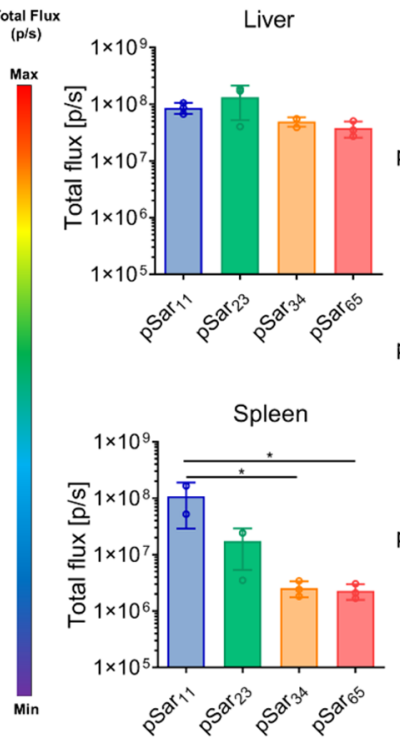

C

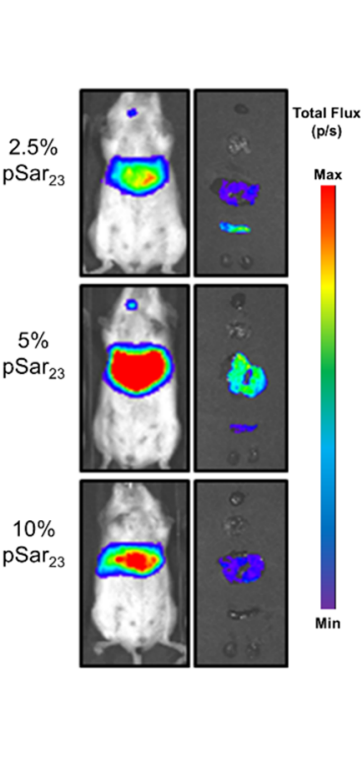

D

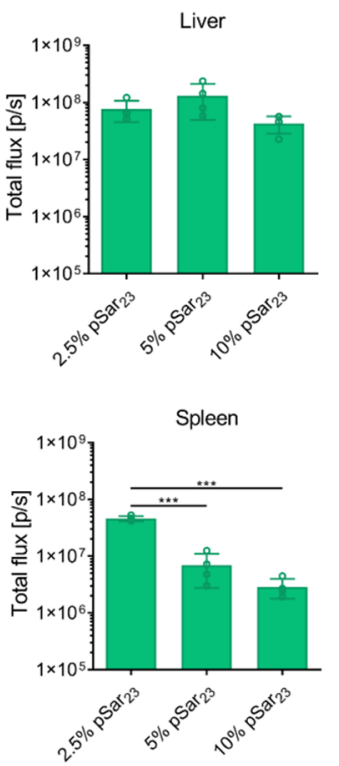

Figure 5. Firefly luciferase expression in Balb/C mice injected intravenously with pSar-LNPs. Transfection at $6 \mathrm{~h}$ post-injection of LNPs with an mRNA dose of $10 \mu \mathrm{g}$. (A) Representative bioluminescence and (B) graphical display of ex vivo luciferase expression in the liver and spleen upon injection with LNPs comprising 5\% pSar lipid with increasing chain length (11-65 sarcosine units). Data are presented as the mean of total flux (photons/second $(\mathrm{p} / \mathrm{s})) \pm$ standard deviation for $n=3$. (C) Representative bioluminescence and (D) graphical display of ex vivo luciferase expression in the liver and spleen upon injection with $\mathrm{pSar}_{23}$ LNPs comprising increased molar fraction (2.5-10\%). Data are presented as the mean of total flux $(\mathrm{p} / \mathrm{s}) \pm$ standard deviation, $n=4$. Statistical significance was calculated with one-way ANOVA with multiple comparisons $(* p<0.05$; $* * p<0.01 ; * * * p<0.001)$

different therapeutic applications, respectively, the information on the structural differences between the two systems might help for tailoring further optimized delivery systems for a given therapeutic intervention.

Transfection of pSar LNPs In Vitro. To investigate the pSar LNP potency, mRNA expression was evaluated in vitro using a hepatocarcinoma cell line (HepG2). For this purpose, LNPs comprising firefly luciferase-encoding mRNA were assembled using either pSar or PEG lipids. Figure 4 shows the luciferase expression $24 \mathrm{~h}$ post-incubation with LNPs manufactured with pSar lipids of different chain lengths or with the PEGylated lipid as a function of their molar fraction. In absolute numbers, the luciferase expression with the pSar systems was lower than with PEGylated systems; however, remarkably, for the pSar LNPs, the luciferase expression increased with increasing pSar lipid content, as can be most clearly seen for the $\mathrm{pSar}_{23}$ formulations (Figure 4A). In contrast, the activity of PEGylated systems decreased with increased PEG lipid fraction. The reduction of luciferase expression with increasing PEG fractions is in accordance with the literature, where the increased stealthy effect of PEG has been correlated with reduced cellular uptake and processing. ${ }^{31}$ The increase of activity with the pSar fraction could be a result of the chemical nature of this grafting moiety, where the secondary amine as an end group might facilitate the electrostatic binding with negatively charged cell membranes. The general observation that for pSar the activity increased with an increasing molar fraction may open novel opportunities for the combination of particle engineering and optimization of biological activity: along with increasing pSar fraction particles size decreases. Small particles can be manufactured without loss of activity as induced by the high fraction of grafted lipid. Chemical modification of the pSar lipids (e.g., with respect to the number and length of hydrocarbon chains or pSar end groups) may allow to further extend the nanoparticle design space and the biological activity of the delivery systems.

Performance of pSar LNPs In Vivo. To investigate the ability of pSar LNPs to safely and efficiently deliver the mRNA in vivo, Balb/C mice were intravenously injected with $10 \mu \mathrm{g}$ of luciferase-encoding mRNA formulated into pSar LNPs. The whole animal and extracted organs were analyzed for bioluminescence. In the primary experiments, the fraction of pSar lipid in the LNPs was kept constant at $5 \mathrm{~mol} \%$ whilst the chain length of pSar was varied from 11 to 65 units. As shown in Figure 5A, 6 h post-injection, all pSar LNPs mediated high mRNA expression in the liver and spleen. Differences in the targeting selectivity and efficacy for the different pSar chain lengths were observed. While with $\mathrm{pSar}_{11}$ the luciferase signal from the spleen was the highest, the signal decreased monotonously with increasing the chain length, and the targeting selectivity shifted toward the liver (Figure 5B). For modulation of the spleen signals, also particle size may have played a role. As reported above, the particle size decreased with increasing pSar chain length (Figure 1), with the $\mathrm{pSar}_{11}$ LNPs being substantially larger than those with a higher chain length. Large particles are known to target more efficiently toward the spleen. ${ }^{3}$ For liver expression, here no such strong modulation was observed, although small particles are considered to be preferential for liver targeting. ${ }^{48}$ Further reduction of the particle size may be necessary to induce stronger effects on the liver signal.

Based on efficacy, the $\mathrm{pSar}_{23}$ was selected for further investigation, where the molar fraction of pSar lipid in the LNPs was varied from 2.5 to $10 \%$. As previously shown for chain length, the molar fraction also has an impact on the 


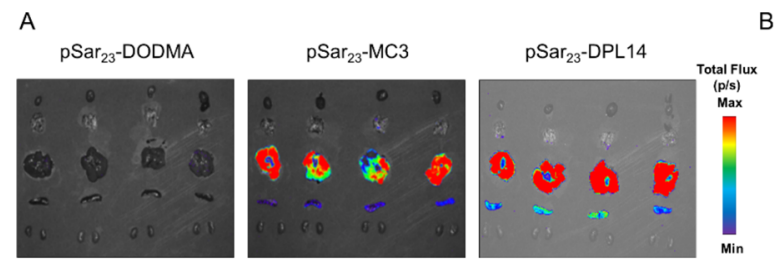

C
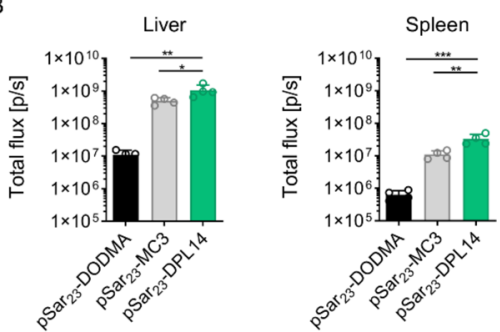

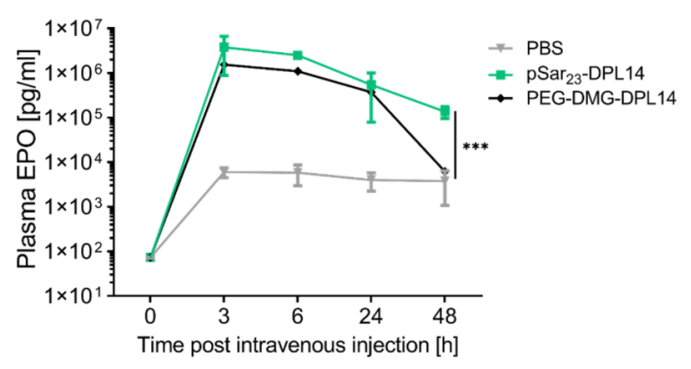

Figure 6. pSar LNP potency with different ionizable lipids. (A) Representative bioluminescence images at $6 \mathrm{~h}$ post-injection of pSar-LNPs comprising the ionizable lipids DODMA, Dlin-MC3-DMA, and DPL14 with $2 \mu \mathrm{g}$ of luciferase mRNA each. (B) Luciferase expression in the liver and spleen. Data are presented as the mean of total flux $(\mathrm{p} / \mathrm{s}) \pm$ standard deviation, $n=4$. (C) Expression profile of EPO plasma concentrations after intravenous injection of $3 \mu \mathrm{g}$ of EPO mRNA-loaded into DPL14-based LNP comprising $5 \%$ pSar 23 or $1.5 \%$ PEG-DMG, mean \pm standard deviation, $n=5$. Statistical significance was calculated with two-way ANOVA with multiple comparisons $(* p<0.05$; $* * p<0.01$; $* * *<0.001)$

biodistribution of the protein expression (Figure 5C,D). Formulations with $2.5 \% \mathrm{pSar}_{23}$ resulted in an increased expression in the spleen, likely caused by a larger particle size $(135 \mathrm{~nm})$, whereas $10 \% \mathrm{pSar}_{23}$ exhibited a specific expression toward the liver as a result of a small particle size $(75 \mathrm{~nm})$, reducing then off-target effects. Thus, the particles can be engineered toward optimized targeting selectivity and activity in the liver and, potentially, other organs (e.g., spleen). Additionally, LNPs comprising 5\% $\mathrm{pSar}_{23}$ resulted in the highest expression in the liver (Figure 5D). While the transfection efficiency of optimized formulations reached the level of PEGylated LNPs (Supporting Figure 3), it appears important to investigate differences in safety and biomarker expression. The potential systemic toxicity of pSar LNPs was addressed through the analysis of global toxicity markers such as serum liver enzymes. Alanine aminotransferase (ALT), aspartate transaminase (AST), and lactate dehydrogenase $(\mathrm{LDH})$ concentrations in the serum were determined $6 \mathrm{~h}$ after a single administration of LNPs $(n=3 /$ group $)$. Supporting Figures 4 and 5 show that only $\mathrm{pSar}_{11}$ and lower molar fractions induced a significant release of liver enzymes above the reference levels obtained for healthy mice, possibly associated with low density of pSar lipid. As shown in Supporting Figure 6, $\mathrm{pSar}_{23}$ LNPs and PEG-DMG induced similar levels of complement $\mathrm{C} 3 \mathrm{a}$ at theoretical plasma concentrations in vitro. However, at higher doses (5X), pSarbased LNPs resulted in substantially lower complement activation when compared to PEG-DMG LNPs.

This data confirm that pSar lipids might allow the assembly of potent lipid nanoparticles for intravenous mRNA delivery with an improved safety profile. The particles can be engineered toward optimized targeting selectivity and activity in the liver and, potentially, other organs (e.g., spleen). pSarcosinylated nanocarriers seem to be less immunogenic than PEGylated systems of comparable composition. Hence, pSar formulations are promising for RNA delivery in settings where immunogenicity needs to be avoided or precisely controlled by the mRNA itself.

Improved Liver Expression from pSar LNPs with Novel Ionizable Lipid. Based on the above-described optimized pSar LNP technology, other ionizable lipids were used for the assembly of the pSar LNPs, with the aim to further improve the overall therapeutic efficacy. The ionizable cationic lipid, DPL14, recently reported for siRNA delivery, as well as the FDA-approved Dlin-MC3-DMA, were used to formulate pSar LNPs. ${ }^{2,49}$ The LNPs from the novel lipids have been demonstrated to be stable and appropriate in all aspects for intravenous application of the mRNA (Supporting Table 3). The in vivo expression profile of the pSar-LNPs is shown in Figure 6. For all ionizable lipids, the luciferase expression was the highest in the liver and spleen (Figure 6A). Here, the luciferase signals from pSar LNPs comprising either DPL14 or Dlin-MC3-DMA were significantly higher than those with DODMA (Figure 6B). LNPs with DPL14 mediated the highest signal, with up to 100 -fold increase in liver expression when compared to DODMA, and up to 2- to 4-fold higher compared to Dlin-MC3-DMA.

For the ionizable lipid with the highest signals, DPL14, the activity of LNPs with $\mathrm{pSar}_{23}$ and PEG was directly compared. Supporting Table 4 summarizes the physicochemical characteristic of both systems. As previously observed for DODMA, pSarcosinylated and PEGylated LNP containing DPL14 promoted comparable luciferase expression in vivo (Supporting Figure 7). To further analyze the potency of pSar LNPs, mRNA encoding a secreted protein erythropoietin (EPO) was formulated and intravenously injected. Remarkably, in that case, pSar $_{23}$ LNPs showed higher and prolonged EPO secretion compared to PEG-DMG LNPs (Figure 6C), although, as shown above, the luciferase expression from PEG-DMG and $\mathrm{pSar}_{23}$ LNPs was similar. The higher durability of the EPO secretion for the pSar LNPs may be related to differences in cellular uptake and processing of these two LNP types, where the lower toxicity of the pSar systems compared 

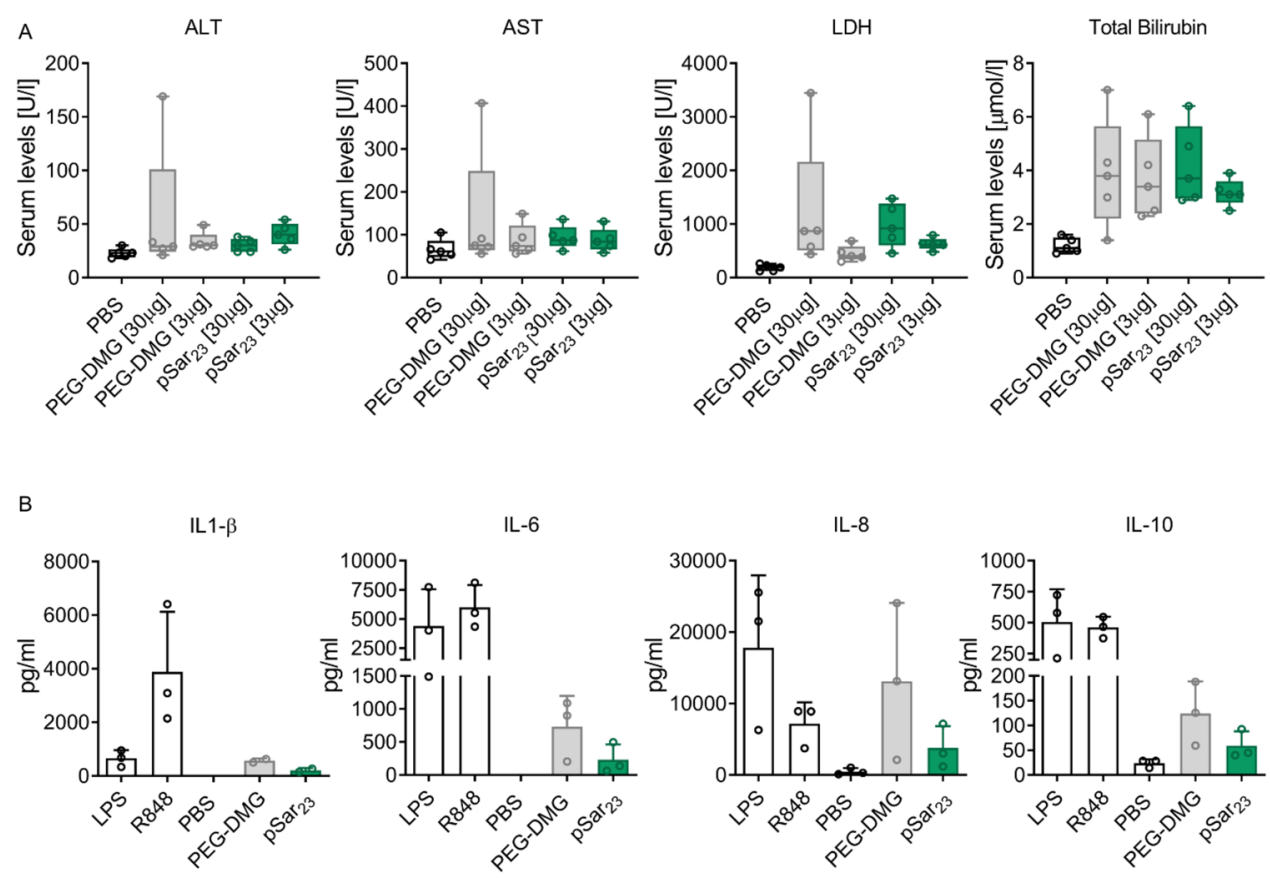

Figure 7. Comparison of the expression and safety profile of pSar and PEG LNPs comprising DPL14 as an ionizable lipid. (A) Liver enzyme release profile upon multiple intravenous injections (total of four weekly injections) of 3 and $30 \mu \mathrm{g}$ of mRNA-LNPs. Measurements were performed $48 \mathrm{~h}$ after last injection, mean \pm standard deviation for $n=5$. (B) Human plasma cytokine profile in whole blood. Cytokines were analyzed using blood from three donors. The shown analytes include IL- $1 \beta$, IL-6, IL-8, and IL-10. The other cytokines (IL-4, IL-5, IL-2, GM-CSF, IFN- $\gamma$, and TNF- $\alpha$ ) showed either overall low expression or minor changes upon treatment (data not shown). Data shown as mean \pm standard deviation.

to PEG systems may be of significance. In contrast to EPO, luciferase expression is resulting from enzymatic amplification that may lead to a misinterpretation of the expression results. Therefore, although the difference between pSar and PEG systems was not very high and most notable only at the later time points, pSar LNPs may have the potential to be even superior to PEGylated LNPs.

Safety Profile of pSarcosinylated LNPs. Toxicity and immunostimulation can be a limiting factor for clinical translation of LNPs comprising novel components. Therefore, we investigated the safety profile (e.g., AST, ALT, LDH, and total bilirubin) of $\mathrm{pSar}_{23}$-DPL14 LNPs in comparison to their PEGylated counterparts in mice models after intravenous administration upon weekly multiple injections at high and low therapeutic doses for 4 weeks (Figure 7A). An increase in the release of liver enzymes for all LNPs compared to the vehicle buffer, $48 \mathrm{~h}$ after last injection, can be observed. Notably, pSarbased LNPs induced similar or lower enzyme levels when compared with the PEG-LNP counterpart. The body weight did not alter upon multiple injections (data not shown). Furthermore, the risk of activating the innate immune system was examined with an in vitro human cytokine release model. Whole blood from three healthy human donors was incubated with mRNA formulated LNPs, including the positive controls lipopolysaccharides (LPS) and resiquimod (R-848). Plasma was collected after $24 \mathrm{~h}$ and analyzed using a Cytokine 10-Plex Human Panel.

Analytes included IL-8, IL-6, IL1- $\beta$, INF- $\gamma$, TNF- $\alpha$, IL-2, IL10 , IL-4, IL-5, and GM-CSF. A selection of four analytes (IL$1 \beta$,IL-6, IL-8, and IL-10) is shown in Figure 7B. The other analyzed cytokines showed either overall low expression or only minor changes upon treatment (data not shown). LNPs formulated with $\mathrm{pSar}_{23}$ showed a reduced cytokine induction when compared with those prepared with PEG-DMG.
Increasing the PEG fraction could also reduce cytokine secretion; however, this is coupled with a decrease of LNP potency. ${ }^{31}$ This very good safety profile of the pSar LNPs, combining reduced hepatic and immune toxicity, together with their high potency in combination with improved ionizable lipids, make them a promising technology for clinical development of RNA therapeutics combining reduced hepatic and immune toxicity and improved activity. Further studies, like investigation of the tolerability in other animal models like rat or non-human primates, will be necessary in order to fully elucidate the applicability of the pSar LNPs as RNA delivery systems with improved safety profile.

\section{CONCLUSIONS}

The development of effective and safe lipid-based formulations for the delivery of mRNA is still one of the biggest challenges for nucleic acid therapy. Here, the polypeptoid pSar, functionalized with a lipophilic anchor, was used to assemble improved LNPs for mRNA delivery. pSar lipids enabled accurate particle engineering through variation of chain length and molar fraction. Depending on the particle characteristics, potent spleen or liver signals were achieved. In combination with novel ionizable lipids, protein secretion in the liver was further improved, while toxicity was reduced when compared to LNPs formulated with PEG-DMG. Furthermore, the reduced cytokine secretion in an in vitro human whole blood model indicated lower immunogenicity of pSarcosinylated LNPs. These findings make pSar-based nanoparticle technology promising for the development of next-generation potent and safe RNA therapeutics for clinical translation.

\section{METHODS}

Materials. Cholesterol and distearoyl-sn-glycerol-3-phosphocholine (DSPC) were from Sigma-Aldrich (St. Louis, MO, USA). The 
ionizable cationic lipid 1,2-dioleyloxy-3-dimethylaminopropane (DODMA) was from Merck (Darmstadt, Germany), and the PEGylated lipid 1,2-dimyristoyl-rac-glycero-3-methylpolyoxyethylene (SUNBRIGHT GM-020(DMG-PEG)) was from NOF (Frankfurt, Germany). The lipid (6Z,9Z,28Z,31Z)-heptatriaconta-6,9,28,31-tetraen-19-yl]4-(dimethylamino)butanoate (Dlin-MC3-DMA) was synthesized by NucleoSyn (Olivet, France). The pSar lipids were either synthesized by the Matthias Barz group (Johannes-Gutenberg Universität Mainz) or from PTS (Valencia, Spain). DPL14 was either synthesized by the Dan Peer Lab (University of Tel Aviv, Israel) or purchased from NucleoSyn (Olivet, France). The EPO and luciferase mRNA was synthesized using 1-methyl-pseudouridine instead of uridine, as described elsewhere. ${ }^{50}$

Lipid Nanoparticle Formulation. Lipid nanoparticles were prepared by mixing an aqueous phase $(0.15 \mathrm{mg} / \mathrm{mL}$ mRNA diluted in $0.1 \mathrm{M}$ citrate buffer, $\mathrm{pH} 5.4$ ) and an organic phase (a lipid mixture of ionizable lipid:phospholipid:cholesterol:stealth component dissolved in ethanol at a molar fraction of 40:50 - X:10:X, respectively, with a total concentration of $13.5 \mathrm{mM}$ ) at a $3: 1$ volume ratio and 12 $\mathrm{mL} / \mathrm{min}$, using a microfluidic instrument (Precision NanoSystems, Vancouver, Canada). The mixture was dialyzed against $1 \mathrm{x}$ DPBS (GIBCO) for $2.5 \mathrm{~h}$ in a Slide-A-Lyser 10K MWCO dialysis cassette (Thermo Fisher Scientific, Waltham, MA, USA) and then concentrated using Amicon 30K Ultra Centrifugal Filters (Merck, Darmstadt, Germany). Time and conditions for dialysis have been proven to be appropriate from internal LNP development experiments. In these experiments, residual ethanol was regularly controlled by osmolality measurements.

Lipid Nanoparticle Characterization. Particle size analysis was determined by dynamic light scattering using a DynaPro Plate Reader II (Wyatt, Dernbach, Germany). From the measurements, size ( $Z$ average), and polydispersity indices (PdI) were calculated from the cumulant analysis using Dynamics 7.8.1.3 software. mRNA assessment was performed by a modified Quant-iT Ribogreen Assay (Invitrogen, Carlsbad, CA). LNP samples or buffer were diluted in $1 \mathrm{x}$ TE buffer to a mRNA concentration between 2 and $5 \mathrm{ng} / \mu \mathrm{L}$. Accessible mRNA was measured by diluting the sample in $1 \mathrm{x}$ TE, and the total RNA amount was quantified by diluting the sample in $2 \%$ Triton X-100 (VWR International GmbH, Darmstadt, Germany). Ribogreen reagent was added to each sample, and the fluorescent signal was quantified in an Infinite F200PRO microplate reader (Tecan, Männedorf, Switzerland). RNA in solution was then assessed by agarose gel electrophoresis using $1 \%$ agarose gel (PanReac AppliChem, Darmstadt, Germany) containing $0.05 \%$ sodium hypochlorite (Sigma-Aldrich Chemie GmbH, Darmstadt Germany), 1x TAE Buffer (Rotiphorese 50X TAE, Carl Roth, Karlsruhe, Germany), and $10 \mu \mathrm{L}$ of GelRed Nucleic Acid Gel Stain (Biotium, Hayward, CA, USA). The LNP samples and naked mRNA were loaded into the gel, which was run at $80 \mathrm{~V}$ for $40 \mathrm{~min}$ and visualized using a Chemidoc XRS imaging system (Bio-Rad, Berkeley, CA, USA).

Small-Angle X-ray Scattering (SAXS). SAXS measurements were conducted at the EMBL P12 bioSAXS beamline at PETRA III, DESY (German Electron Synchrotron, Hamburg, Germany). The measurements were conducted at an X-ray energy of $10 \mathrm{keV}$ and a flux of $5 \times 10^{12} \mathrm{ph} / \mathrm{s}$; the beam size at the sample position was $0.2 \times 0.3$ $\mathrm{mm}^{2}(v \times h$, full width half-maximum, FWHM) utilizing the BioSAXS Sample Changer. ${ }^{51}$ Low volumes $(30 \mu \mathrm{L})$ of the sample were transferred to a quartz capillary mounted in vacuum, and a PILATUS $6 \mathrm{M}$ detector was used to collect images. Images of the scattering signal were processed by the SASFLOW pipeline: ${ }^{52}$ all images were radially averaged, and the frames were compared for radiation damage and excluded from analysis if subjected to radiation damage. Images were averaged and imported into Origin 9.1. Blank references were used to subtract the background scattering from the sample measurements.

X-ray Data Analysis. X-ray scattering data give information on the electron density profile inside the scattering units, here the RNA lipid nanoparticles. The scattering intensity, $I(q)$, as a function of the momentum transfer, $q$, is directly correlated to the autocorrelation function of the electron density distribution, $\rho(r)$ :

$$
I(q) \sim\left|\int \rho(r) \mathrm{e}^{i q r} d^{3} r\right|^{2}
$$

The scattering vector $q$ is given as $q=4 \pi / \lambda \sin (2 \Theta / 2)$, with the $\mathrm{X}$ ray wavelength $\lambda$ and the scattering angle $2 \Theta$. The characteristic length information scales with $1 / q$, i.e., scattering at a low $q$ gives information on large sizes, while the scattering at a high $q$ comprises information on smaller sizes. For the data observed here, with the measured $q$ range from about 0.03 to about $5 \mathrm{~nm}^{-1}$, scattering from structures at length scales between several tens of nanometers down to the angstrom range becomes relevant, where information on ordered and non-ordered structures can be derived. In case repeating stacks of defined structures (such as bilayers) are present, the scattering intensity can be approximated as a function of the particle form factor, $F(q)$, and the structure factor $G(q)$ :

$$
I(q) \approx F(q) \times G(q)
$$

The structure factor represents repeating patters with defined organization, such as outlined below. The form factor derives from the electron density distribution of single or non-ordered scattering units, which can be the whole particle, or as well the singe bilayer in a stack. For example, for spherical compact particles, a scattering curve with oscillations according to

$$
I(q) \approx\left[\frac{3[\sin (q \times R)-q \times R \times \cos (q \times R)]}{(q \times R)^{3}}\right]^{2}
$$

would be expected (where $q$ is the momentum transfer and $R$ is the radius of the particle). This curve has a first minimum at $q=4.66 / R$. This formalism can be used to get an estimate on the particle size, neglecting parameters like polydispersity, anisotropy, and surface roughness of packing compactness.

Some further information on the particle characteristics can be obtained by analyzing the intensity decay using the power law

$$
I(q) \approx I(0) \times q^{-x}
$$

which provides insight into the packing compactness and fractal dimensionality of the particles. For particles with smooth surfaces, the intensity decays with the fourth power of $q$ (Porod law), where the decay becomes less steep if the roughness increases. For example, a rough interface is expected to yield a value of 3 for the exponent, while for Gaussian chains, a value of 2 is expected (also flat particles or increased polydispersity can have this effect). ${ }^{53}$

Scattering from ordered structures results in peaks, from which further information on the internal organization can be derived. Using Bragg's law, the repeat distance is obtained from the peak position according to

$$
d=\frac{n \times 2 \pi}{q_{n}}
$$

where $q_{n}$ is the maximum position of the $n$th order peak. Ordered stacks of lipid based aggregates comprising nucleotides can be present with different types of organization, depending on the manufacturing protocol, temperature, type of lipid components, and other parameters. Typical types of organization are lamellar stacks, resulting in equidistant Bragg peaks (positions 1, 2, 3, ...), hexagonal structures (positions $1, \sqrt{3}, 2, \sqrt{7}, 3)$, or cubic phases $(1, \sqrt{2}, \sqrt{3}, 2, \sqrt{5}, \ldots) .{ }^{54}$ Since here, basically, only one and very broad peak $(n=1)$ was present, a discrimination between these types of organization is not meaningful because the number of repeat units in the stacks is too low. Here, the width, $w$, of the peaks, was analyzed in order to get information on the number or ordered units or, more generally speaking, on the positional order of the stacks. Lipidic systems in an aqueous environment are usually being described as liquid crystalline phases, where the positional order decays as a function of the distance. In a generally accepted model to describe liquid crystalline ${ }^{34}$ 
arrays, the correlation length, $\xi$, defined as the distance, at which the positional correlation decays to the value $1 / e$ is given as

$$
\xi \approx \frac{2}{w}
$$

The area of the peaks was taken as a measure for the total amount of material present in the respective state of organization. The high peak widths and thus the low correlation lengths observed here indicate that only a low single digit number of ordered units was present in the stacks. Therefore, as a further a test for plausibility, the scattering curves were compared with diffraction patterns from organized units using basic formalisms in in an utmost reductionist manner. One-dimensional ordered arrays consisting of scatterers of homogeneous internal electron density distribution and fixed length, $l$, forming stacks with spacing, $d$, where scattering for increasing numbers, $N(N=2,3,4, \ldots)$, in the stacks was calculated. Following eq 2 , for the form factor, a slit function was used:

$$
F(q) \approx\left|\frac{\sin \left(q \times \frac{l}{2}\right)}{q \times \frac{l}{2}}\right|^{2}
$$

and for the structure factor, a lattice with $N$ repeat units is considered:

$$
G(q) \approx\left|\frac{\sin \left(N \times q \times \frac{d}{2}\right)}{q \times \frac{d}{2}}\right|^{2}
$$

Simulations and data analysis were performed with the OriginLab Corporation (Northampton, MA, USA) software package. The implemented non-linear fitting protocol was used for the fitting of Lorentzian peak profiles using the formalism

$$
y=\frac{2 \times A}{\pi} \times \frac{w}{4 \times\left(q-q_{c}\right)^{2}+w^{2}}
$$

to obtain peak position, $q_{0}$ peak width, $w$, and peak area, $A$, from the experimental data as shown in the text. A linear baseline was subtracted from the data manually prior to fitting. In certain cases, double Lorentzian fitting was tried to model the data. Due to the large width of the main peaks, there was an indication that these should be better represented by the superposition of two separate peaks such as resulting from organized stacks of pure lipids and lipid complexed RNA with different spacings. With that, lower values of $\chi^{2}$ were obtained; however, no substantial additional information was obtained, and therefore, for most cases, only single Lorentzian was used. Only in the case of the systems with high fractions of $\mathrm{pSar}_{11}$, where the presence of two peaks was obvious, that both peaks were fitted.

Cell Culture. HepG2 cells were grown in EMEM (Eagle's minimum essential smedium, ATCC) supplemented with $10 \%$ noninactivated FCS (fetal calf serum, Biochrom, Berlin, Germany) at 37 ${ }^{\circ} \mathrm{C}$ and $5 \% \mathrm{CO}_{2}$ environment and subcultured using StemPro Accutase (Thermo Fisher Scientific, Waltham, MA, USA). HepG2 cells were seeded at 20,000 cells/well in a Nunc white flat-bottom 96well plate (Merck KGaA, Darmstadt, Germany) and allowed to grow for $24 \mathrm{~h}$ prior to transfection. Formulations were added at mRNA concentration at a final dose of $25 \mathrm{ng}$ mRNA per well with a total volume of $100 \mu \mathrm{L}$. After $24 \mathrm{~h}$, luciferase expression (Bright-Glo Luciferase assay, Promega, Madison, WI, USA) and cell viability (Cell Titer-Glo assay, Promega, Madison, WI, USA) were performed according to the manufacturer's guidelines.

Animal Experiments. Healthy female Balb/C mice were obtained from Charles River. All mice were kept following the federal and state policies on animal research at BioNTech SE. For biodistribution studies, lipid nanoparticles were intravenously administered through retro-orbital sinus injection at an mRNA dose of 10 or $2 \mu \mathrm{g}$. After $6 \mathrm{~h}$, mice were administered intraperitoneally with D-luciferin solution (Becton Dickinson $\mathrm{GmbH}$ Biosciences, Heidelberg, Germany) at $100 \mathrm{mg} / \mathrm{kg}$ body weight. Bioluminescence was performed on mice or isolated organs using an Xenogen IVIS Spectrum (Caliper Life Sciences, Hopkinton, MA, USA) within 10$25 \mathrm{~min}$ of luciferin administration. Images were quantified by region of interest for total flux using Living Image Software (Perkin Elmer). For EPO experiments, mice received a single intravenous injection of mRNA-loaded LNPs at RNA concentrations of 30 and $3 \mu \mathrm{g}$. After 3, 6,24 , and $48 \mathrm{~h}$, a minimal blood volume $(18 \mu \mathrm{L})$ was withdrawn and the plasma was obtained by centrifuging the whole blood at the $13,000 \mathrm{~g}$ for $3 \mathrm{~min}$. EPO levels were determined using a Mouse Erythropoietin/EPO DuoSet ELISA kit (R\&D Systems, Abingdon, UK) following the manufacturer's suggestions. For toxicological studies, the mice received multiple injections, weekly, at an mRNA dose of 30 and $3 \mu \mathrm{g}$. At $48 \mathrm{~h}$ after the last injection, blood was drawn, and the serum was obtained by centrifuging the whole blood for $3 \mathrm{~min}$ at $10,000 \mathrm{~g}$. EPO levels were determined using a Mouse Erythropoietin/EPO DuoSet ELISA kit (R\&D Systems, Abingdon, UK) following the manufacturer's suggestions. Liver enzyme levels of alanine aminotransferase (ALT), aspartate aminotransferase (AST), lactate dehydrogenase ( $\mathrm{LDH})$, and total bilirubin levels were determined using Indiko (Thermo Fisher Scientific, Waltham, Massachusetts, United States).

Cytokines Release. Human whole blood from three healthy donors was collected with a Li-heparin vacutainer tube (BD Vacutainer Li-heparin, Becton Dickinson GmbH, Heidelberg, Germany). Whole blood was diluted 4X with RPMI culture medium (RPMI 1640-GlutaMAX-I, Thermo Fisher Scientific, Waltham, Massachusetts, United States) containing 10\% FBS (heat-inactivated) and plated in a flat-bottom 96-well plate at $180 \mu \mathrm{L} /$ well. Then, $20 \mu \mathrm{L}$ of controls and $20 \mu \mathrm{L}$ of LNPs at a theoretical plasma concentration of $0.0125 \mathrm{mg} / \mathrm{mL}$ (corresponding to $1 \mathrm{mg} / \mathrm{kg}$ ) were added to the whole blood and incubated at $37{ }^{\circ} \mathrm{C}, 5 \% \mathrm{CO}_{2}$ for $24 \mathrm{~h}$. Positive controls such as resiquimod (R-848) (Merck KGaA, Darmstadt, Germany) and LPS (lipopolysaccharide) (Invivogen, San Diego, CA, USA) were added at assay concentrations of $10 \mathrm{mM}$ and $20 \mathrm{ng} / \mathrm{mL}$, respectively. Plasma was then collected and stored at $-80{ }^{\circ} \mathrm{C}$ until analysis. Cytokine levels were measured using a Bio-Pex 200 Luminex (Bio-rad Laboratories, Hercules, CA, USA) with Cytokine Human Ultrasensitive Magnetic 10-Plex Pane (Thermo Fisher Scientific, Waltham, Massachusetts, United States); analytics included the following: GM-CSF, IFN- $\gamma$, IL-1 $\beta$, IL-2, IL-4, IL-5, IL-6, IL-8, IL10 , and TNF- $\alpha$.

Complement Activation. In vitro $\mathrm{C} 3 \mathrm{a}$ levels were determined using the Human C3a EIA kit (Quidel, San Diego, USA). Briefly, samples and positive (Cremophor El, Merck, Darmstadt, Germany) and negative (1x DPBS and EDTA $(18 \mathrm{mM})$ ) controls were incubated with Normal Human Serum Complement (NHS, Quidel, San Diego, USA) at a ratio of 20:80 (Specimen: NHS) for $1 \mathrm{~h}$ at 37 ${ }^{\circ} \mathrm{C}$. LNPs were incubated with human serum at a final mRNA concentration of $0.0125 \mathrm{mg} / \mathrm{mL}$, the theoretical concentration (corresponding to a dose of $1 \mathrm{mg} / \mathrm{kg}$ ), and $0.0625 \mathrm{mg} / \mathrm{mL}$, five times the theoretical human plasma concentration. C3a EIA kit (Quidel, San Diego, USA) was executed according to the manufacturer's protocol.

Data Analysis. All statistical analysis was conducted using GraphPad Prism 8 software. Error bars represent one standard deviation (sample sizes provided in each figure). To compare two groups, a one-way ANOVA test was performed, assuming Gaussian distribution. Groups of three or more were compared by one-way ANOVA. Statistical significance is indicated by ${ }^{*} p<0.05$, $* * p<0.01$, and $* * * * p<0.0001$

\section{ASSOCIATED CONTENT}

\section{(s) Supporting Information}

The Supporting Information is available free of charge at https://pubs.acs.org/doi/10.1021/acsanm.0c01834.

(Supporting Figure 1) General overview of SAXS data from all systems, (Supporting Figure 2) simulation and experimental data in a linear scale, (Supporting Figure 3) 
luciferase expression upon IV injection of PEG LNPs, (Supporting Figure 4) effect of pSar chain length on the liver enzyme release profile, (Supporting Figure 5) effect of pSar molar faction on the liver enzyme release profile, (Supporting Figure 6) concentrations of C3a postincubation of pSar23 and PEG LNPs with human serum, (Supporting Figure 7) luciferase expression of pSar23 and PEG formulations containing DPL14 as the ionizable lipid, (Supporting Table 1) quantitative analysis of the peak as a function of pSar and PEGDMG molar fraction, (Supporting Table 2) quantitative analysis of the peak as a function of pSar and PEG-DMG molar fraction, (Supporting Table 3) physicochemical characterization of pSar23-LNPs comprising DODMA, Dlin-MC3-DMA, and DPL14 ionizable cationic lipids, and (Supporting Table 4) physicochemical characterization of pSar23 and PEG-DMG LNP containing DPL14 ionizable cationic lipids (PDF)

\section{AUTHOR INFORMATION}

\section{Corresponding Author}

Heinrich Haas - BioNTech RNA Pharmaceuticals GmbH, 55131 Mainz, Germany; 이이.org/0000-0002-55175970; Email: Heinrich.Haas@biontech.de

\section{Authors}

Sara S. Nogueira - BioNTech RNA Pharmaceuticals GmbH, 55131 Mainz, Germany

Anne Schlegel - BioNTech RNA Pharmaceuticals GmbH, 55131 Mainz, Germany

Konrad Maxeiner - BioNTech RNA Pharmaceuticals GmbH, 55131 Mainz, Germany

Benjamin Weber - Institute of Organic Chemistry, Johannes Gutenberg University Mainz, 55128 Mainz, Germany

Matthias Barz - Leiden Academic Center for Drug Research, Leiden University, 2333 CC Leiden, Netherlands; 10 orcid.org/ 0000-0002-1749-9034

Martin A. Schroer - European Molecular Biology Laboratory (EMBL) Hamburg c/o DESY, 22607 Hamburg, Germany; (1) orcid.org/0000-0002-0747-3965

Clement E. Blanchet - European Molecular Biology Laboratory (EMBL) Hamburg c/o DESY, 22607 Hamburg, Germany

Dmitri I. Svergun - European Molecular Biology Laboratory (EMBL) Hamburg c/o DESY, 22607 Hamburg, Germany

Srinivas Ramishetti - Laboratory of Precision NanoMedicine, The Shmunis School of Biomedicine and Cancer Research and Dept. of Materials Sciences and Engineering, Tel Aviv University, Tel Aviv 69978, Israel

Dan Peer - Laboratory of Precision NanoMedicine, The Shmunis School of Biomedicine and Cancer Research and Dept. of Materials Sciences and Engineering, Tel Aviv University, Tel Aviv 69978, Israel; 이이.org/0000-0001-8238-0673

Peter Langguth - Department of Pharmaceutics and Biopharmaceutics, Johannes Gutenberg University Mainz, 55099 Mainz, Germany

Ugur Sahin - BioNTech RNA Pharmaceuticals GmbH, 55131 Mainz, Germany; TRON - Translational Oncology at the University Medical Center of Johannes Gutenberg University Mainz gGmbH, 55099 Mainz, Germany; Research Center for Immunotherapy (FZI), University Medical Center at the Johannes Gutenberg University Mainz, 55099 Mainz, Germany
Complete contact information is available at:

https://pubs.acs.org/10.1021/acsanm.0c01834

\section{Author Contributions}

S.S.N., A.S., and H.H. designed and analyzed the experiments. S.S.N. and performed the experiments. S.S.N. and H.H. wrote the manuscript. The manuscript was written through contributions of all authors. All authors have given approval to the final version of the manuscript.

\section{Notes}

The authors declare no competing financial interest.

\section{ACKNOWLEDGMENTS}

U.S., P.L., and M.B. would like to acknowledge funding by the Deutsche Forschungsgemeinschaft (Collaborative Research Center 1066). A Lewis Trust grant was awarded to Dan Peer. We would like to thank to Janina Kuschmann for the support on physicochemical and in vitro analysis, all the animal groups for the support on the animal experiments, and finally the synthesis unit of BioNTech for the RNA synthesis. We also would like to thank Rachel Xerri for proofreading of the manuscript. We would like to thank Cristina Sala for creating the graphical abstract and the LNP cartoon.

\section{ABBREVIATIONS}

ABC, accelerated blood clearance

ALT, alanine transaminase

AST, aspartate transaminase

CARPA, complement activation-related pseudo-allergy

Dlin-MC3-DMA, O-(Z,Z,Z,Z-heptatriaconta-6,9,26,29-tetraen-19-yl)-4-( $N, N$-dimethylamino)

DLS, dynamic light scattering

DODMA, 1,2-dioleyloxy-3-dimethylaminopropane

DSPC, 1,2-distearoyl-sn-glycero-3-phosphocholine

EMEM, Eagle's minimum essential medium

EPO, erythropoietin

FCS, fetal calf serum

FDA, Food and Drug Administration

$\mathrm{LDH}$, lactate dehydrogenase

LNPs, lipid nanoparticles

LPS, lipopolysaccharides

mRNA, messenger RNA

$\mathrm{N} / \mathrm{P}$, nitrogen to phosphate

PdI, polydispersity index

PEG, polyethylene glycol

PEG-DMG, 1,2-dimyristoyl-rac-glycero-3-methylpolyoxyethylene

pSar, polysarcosine

SAXS, small-angle X-ray scattering

siRNA, short interfering RNA

\section{REFERENCES}

(1) Sahin, U.; Karikó, K.; Türeci, Ö. MRNA-Based Therapeutics Developing a New Class of Drugs. Nat. Rev. Drug Discovery 2014, 13, $759-780$.

(2) Kowalski, P. S.; Rudra, A.; Miao, L.; Anderson, D. G. Delivering the Messenger: Advances in Technologies for Therapeutic MRNA Delivery. Mol. Ther. 2019, 27, 710-728.

(3) Kranz, L. M.; Diken, M.; Haas, H.; Kreiter, S.; Loquai, C.; Reuter, K. C.; Meng, M.; Fritz, D.; Vascotto, F.; Hefesha, H.; Grunwitz, C.; Vormehr, M.; Hüsemann, Y.; Selmi, A.; Kuhn, A. N.; Buck, J.; Derhovanessian, E.; Rae, R.; Attig, S.; Diekmann, J.; Jabulowsky, R. A.; Heesch, S.; Hassel, J.; Langguth, P.; Grabbe, S.; 
Huber, C.; Türeci, Ö.; Sahin, U. Systemic RNA Delivery to Dendritic Cells Exploits Antiviral Defence for Cancer Immunotherapy. Nature 2016, 534, 396-401.

(4) Feldman, R. A.; Fuhr, R.; Smolenov, I.; Ribeiro, A.; Panther, L.; Watson, M.; Senn, J. J.; Smith, M.; Almarsson, Ö.; Pujar, H. S.; Laska, M. E.; Thompson, J.; Zaks, T.; Ciaramella, G. MRNA Vaccines against H10N8 and H7N9 Influenza Viruses of Pandemic Potential Are Immunogenic and Well Tolerated in Healthy Adults in Phase 1 Randomized Clinical Trials. Vaccine 2019, 37, 3326-3334.

(5) Grabbe, S.; Haas, H.; Diken, M.; Kranz, L. M.; Langguth, P.; Sahin, U. Translating Nanoparticulate-Personalized Cancer Vaccines into Clinical Applications: Case Study with RNA-Lipoplexes for the Treatment of Melanoma. Nanomedicine 2016, 11, 2723-2734.

(6) Semple, S. C.; Klimuk, S. K.; Harasym, T. O.; Santos, N. D.; Ansell, S. M.; Wong, K. F.; Maurer, N.; Stark, H.; Cullis, P. R.; Hope, M. J.; Scherrer, P. Efficient Encapsulation of Antisense Oligonucleotides in Lipid Vesicles Using Ionizable Aminolipids: Formation of Novel Small Multilamellar Vesicle Structures. Biochim. Biophys. Acta, Biomembr. 2001, 1510, 152-166.

(7) Akinc, A.; Maier, M. A.; Manoharan, M.; Fitzgerald, K.; Jayaraman, M.; Barros, S.; Ansell, S.; Du, X.; Hope, M. J.; Madden, T. D.; Mui, B. L.; Semple, S. C.; Tam, Y. K.; Ciufolini, M.; Witzigmann, D.; Kulkarni, J. A.; van der Meel, R.; Cullis, P. R. The Onpattro Story and the Clinical Translation of Nanomedicines Containing Nucleic Acid-Based Drugs. Nat. Nanotechnol. 2019, 14, 1084-1087.

(8) Evers, M. J. W.; Kulkarni, J. A.; van der Meel, R.; Cullis, P. R.; Vader, P.; Schiffelers, R. M. State-of-the-Art Design and Rapid-Mixing Production Techniques of Lipid Nanoparticles for Nucleic Acid Delivery. Small Methods 2018, 2, 1700375.

(9) Ishida, T.; Masuda, K.; Ichikawa, T.; Ichihara, M.; Irimura, K.; Kiwada, H. Accelerated Clearance of a Second Injection of PEGylated Liposomes in Mice. Int. J. Pharm. 2003, 255, 167-174.

(10) Ishida, T.; Ichihara, M.; Wang, X.; Yamamoto, K.; Kimura, J.; Majima, E.; Kiwada, H. Injection of PEGylated Liposomes in Rats Elicits PEG-Specific IgM, Which Is Responsible for Rapid Elimination of a Second Dose of PEGylated Liposomes. J. Controlled Release 2006, $112,15-25$.

(11) Semple, S. C.; Harasym, T. O.; Clow, K. A.; Ansell, S. M.; Klimuk, S. K.; Hope, M. J. Immunogenicity and Rapid Blood Clearance of Liposomes Containing Polyethylene Glycol-Lipid Conjugates and Nucleic Acid. J Pharmacol Exp Ther 2005, 312, $1020-1026$.

(12) Garay, R. P.; Labaune, J. P. Immunogenicity of Polyethylene Glycol (PEG). In The Open Conference Proceedings Journal; 2011; 2, 104-107, DOI: $10.2174 / 2210289201102010104$.

(13) Chen, B.-M.; Su, Y.-C.; Chang, C.-J.; Burnouf, P.-A.; Chuang, K.-H.; Chen, C.-H.; Cheng, T.-L.; Chen, Y.-T.; Wu, J.-Y.; Roffler, S. R. Measurement of Pre-Existing IgG and IgM Antibodies against Polyethylene Glycol in Healthy Individuals. Anal. Chem. 2016, 88, 10661-10666.

(14) Szebeni, J.; Muggia, F.; Gabizon, A.; Barenholz, Y. Activation of Complement by Therapeutic Liposomes and Other Lipid ExcipientBased Therapeutic Products: Prediction and Prevention. Adv Drug Deliver Rev. 2011, 63, 1020-1030.

(15) Szebeni, J. Complement Activation-Related Pseudoallergy: A Stress Reaction in Blood Triggered by Nanomedicines and Biologicals. Mol. Immunol. 2014, 61, 163-173.

(16) Knop, K.; Hoogenboom, R.; Fischer, D.; Schubert, U. S. Poly(Ethylene Glycol) in Drug Delivery: Pros and Cons as Well as Potential Alternatives. Angew. Chem., Int. Ed. 2010, 49, 6288-6308.

(17) Abbina, S.; Parambath, A. 14 PEGylation and Its Alternatives A Summary. In Engineering of Biomaterials for Drug Delivery Systems; 2018; 363-376. DOI: 10.1016/b978-0-08-101750-0.00014-3.

(18) Gangloff, N.; Ulbricht, J.; Lorson, T.; Schlaad, H.; Luxenhofer, R. Peptoids and Polypeptoids at the Frontier of Supra- and Macromolecular Engineering. Chem. Rev. 2016, 116, 1753-1802.

(19) Secker, C.; Brosnan, S. M.; Luxenhofer, R.; Schlaad, H. Poly $(\alpha-$ Peptoid)s Revisited: Synthesis, Properties, and Use as Biomaterial. Macromol. Biosci. 2015, 15, 881-891.
(20) Kidchob, T.; Kimura, S.; Imanishi, Y. Amphiphilic Poly(Ala)-bPoly(Sar) Microspheres Loaded with Hydrophobic Drug. J. Controlled Release 1998, 51, 241-248.

(21) Fenaroli, F.; Repnik, U.; Xu, Y.; Johann, K.; Van Herck, S.; Dey, P.; Skjeldal, F. M.; Frei, D. M.; Bagherifam, S.; Kocere, A.; Haag, R.; De Geest, B. G.; Barz, M.; Russell, D. G.; Griffiths, G. Enhanced Permeability and Retention-like Extravasation of Nanoparticles from the Vasculature into Tuberculosis Granulomas in Zebrafish and Mouse Models. ACS Nano 2018, 12, 8646-8661.

(22) Birke, A.; Ling, J.; Barz, M. Polysarcosine-Containing Copolymers: Synthesis, Characterization, Self-Assembly, and Applications. Prog. Polym. Sci. 2018, 81, 163-208.

(23) Zimpel, A.; Al Danaf, N.; Steinborn, B.; Kuhn, J.; Höhn, M.; Bauer, T.; Hirschle, P.; Schrimpf, W.; Engelke, H.; Wagner, E.; Barz, M.; Lamb, D. C.; Lächelt, U.; Wuttke, S. Coordinative Binding of Polymers to Metal-Organic Framework Nanoparticles for Control of Interactions at the Biointerface. ACS Nano 2019, 13, 3884-3895.

(24) Negwer, I.; Best, A.; Schinnerer, M.; Schäfer, O.; Capeloa, L.; Wagner, M.; Schmidt, M.; Mailänder, V.; Helm, M.; Barz, M.; Butt, H.-J.; Koynov, K. Monitoring Drug Nanocarriers in Human Blood by Near-Infrared Fluorescence Correlation Spectroscopy. Nat. Commun. 2018, 9, 5306.

(25) Stéen, E. J. L.; Jørgensen, J. T.; Johann, K.; Nørregaard, K.; Sohr, B.; Svatunek, D.; Birke, A.; Shalgunov, V.; Edem, P. E.; Rossin, R.; Seidl, C.; Schmid, F.; Robillard, M. S.; Kristensen, J. L.; Mikula, H.; Barz, M.; Kjær, A.; Herth, M. M. Trans-Cyclooctene-Functionalized PeptoBrushes with Improved Reaction Kinetics of the Tetrazine Ligation for Pretargeted Nuclear Imaging. ACS Nano 2019, 14, 568-584.

(26) Weber, B.; Seidl, C.; Schwiertz, D.; Scherer, M.; Bleher, S.; Süss, R.; Barz, M. Polysarcosine-Based Lipids: From Lipopolypeptoid Micelles to Stealth-Like Lipids in Langmuir Blodgett Monolayers. Polymers 2016, 8, 427.

(27) Weber, B.; Birke, A.; Fischer, K.; Schmidt, M.; Barz, M. Solution Properties of Polysarcosine: From Absolute and Relative Molar Mass Determinations to Complement Activation. Macromolecules 2018, 51, 2653-2661.

(28) Maurer, P. H.; Subrahmanyam, D.; Katchalski, E.; Blout, E. R. Antigenicity of Polypeptides (Poly Alpha Amino Acids). J. Immunol. 1959, 83, 193-197.

(29) Belliveau, N. M.; Huft, J.; Lin, P. J.; Chen, S.; Leung, A. K.; Leaver, T. J.; Wild, A. W.; Lee, J. B.; Taylor, R. J.; Tam, Y. K.; Hansen, C. L.; Cullis, P. R. Microfluidic Synthesis of Highly Potent Limit-Size Lipid Nanoparticles for In Vivo Delivery of SiRNA. Mol. Ther.-Nucleic Acids 2012, 1, No. e37.

(30) Muhl, C.; Conrad, M.; Unthan, D.; Barz, M. Synthesis and Characterization of Bisalkylated Polysarcosine-Based Lipopolymers. Eur. Polym. J. 2019, 120, 109223.

(31) Kumar, V.; Qin, J.; Jiang, Y.; Duncan, R. G.; Brigham, B.; Fishman, S.; Nair, J. K.; Akinc, A.; Barros, S. A.; Kasperkovitz, P. V. Shielding of Lipid Nanoparticles for SiRNA Delivery: Impact on Physicochemical Properties, Cytokine Induction, and Efficacy. Mol. Ther.-Nucleic Acids 2014, 3, e210.

(32) Bale, H. D.; Schmidt, P. W. Small-Angle X-Ray-Scattering Investigation of Submicroscopic Porosity with Fractal Properties. Phys. Rev. Lett. 1984, 53, 596-599.

(33) Teixeira, J. Small-Angle Scattering by Fractal Systems. J. Appl. Crystallogr. 1988, 21, 781-785.

(34) Goodby, J. W.; Tschierske, C.; Gleeson, H.; Kato, T.; Raynes, P.; Collings, P. J. Handbook of Liquid Crystals; 2014; DOI: 10.1002/ 9783527671403.

(35) Siewert, C. D.; Haas, H.; Cornet, V.; Nogueira, S. S.; Nawroth, T.; Uebbing, L.; Ziller, A.; Al-Gousous, J.; Radulescu, A.; Schroer, M. A.; Blanchet, C. E.; Svergun, D. I.; Radsak, M. P.; Sahin, U.; Langguth, P. Hybrid Biopolymer and Lipid Nanoparticles with Improved Transfection Efficacy for MRNA. Cell 2020, 9, 2034.

(36) Leung, A. K. K.; Hafez, I. M.; Baoukina, S.; Belliveau, N. M.; Zhigaltsev, I. V.; Afshinmanesh, E.; Tieleman, D. P.; Hansen, C. L.; Hope, M. J.; Cullis, P. R. Lipid Nanoparticles Containing SiRNA 
Synthesized by Microfluidic Mixing Exhibit an Electron-Dense Nanostructured Core. J. Phys. Chem. C 2012, 116, 18440-18450.

(37) Leung, A. K. K.; Tam, Y. Y. C.; Cullis, P. R. Chapter Four Lipid Nanoparticles for Short Interfering RNA Delivery. Adv. Genet. 2014, $88,71-110$.

(38) Patel, S.; Ashwanikumar, N.; Robinson, E.; Xia, Y.; Mihai, C.; Griffith, J. P., III; Hou, S.; Esposito, A. A.; Ketova, T.; Welsher, K.; Joyal, J. L.; Almarsson, Ö.; Sahay, G. Naturally-Occurring Cholesterol Analogues in Lipid Nanoparticles Induce Polymorphic Shape and Enhance Intracellular Delivery of MRNA. Nat. Commun. 2020, 11, 983.

(39) Kulkarni, J. A.; Darjuan, M. M.; Mercer, J. E.; Chen, S.; van der Meel, R.; Thewalt, J. L.; Tam, Y. Y. C.; Cullis, P. R. On the Formation and Morphology of Lipid Nanoparticles Containing Ionizable Cationic Lipids and SiRNA. ACS Nano 2018, 12, 4787.

(40) Xu, Y.; Hui, S.-W.; Frederik, P.; Szoka, F. C., Jr. Physicochemical Characterization and Purification of Cationic Lipoplexes. Biophys. J. 1999, 77, 341-353.

(41) Salditt, T.; Koltover, I.; Rädler, J. O.; Safinya, C. R. TwoDimensional Smectic Ordering of Linear DNA Chains in SelfAssembled DNA-Cationic Liposome Mixtures. Phys. Rev. Lett. 1997, $79,2582-2585$.

(42) Koltover, I.; Salditt, T.; Safinya, C. R. Phase Diagram, Stability, and Overcharging of Lamellar Cationic Lipid-DNA Self-Assembled Complexes. Biophys. J. 1999, 77, 915-924.

(43) RÄdler, J. O.; Koltover, I.; Salditt, T.; Safinya, C. R. Structure of DNA-Cationic Liposome Complexes: DNA Intercalation in Multilamellar Membranes in Distinct Interhelical Packing Regimes. Science 1997, 275, 810-814.

(44) Balbino, T. A.; Gasperini, A. A. M.; Oliveira, C. L. P.; Azzoni, A. R.; Cavalcanti, L. P.; de La Torre, L. G. Correlation of the Physicochemical and Structural Properties of PDNA/Cationic Liposome Complexes with Their in Vitro Transfection. Langmuir 2012, 28, 11535-11545.

(45) Majzoub, R. N.; Ewert, K. K.; Safinya, C. R. Cationic Liposome-Nucleic Acid Nanoparticle Assemblies with Applications in Gene Delivery and Gene Silencing. Philos. Trans. R. Soc., A 2016, 374, 20150129.

(46) Ziller, A.; Nogueira, S. S.; Hühn, E.; Funari, S. S.; Brezesinski, G.; Hartmann, H.; Sahin, U.; Haas, H.; Langguth, P. Incorporation of MRNA in Lamellar Lipid Matrices for Parenteral Administration. Mol. Pharmaceutics 2018, 15, 642-651.

(47) Safinya, C. R. Structures of Lipid-DNA Complexes: Supramolecular Assembly and Gene Delivery. Curr. Opin. Struct. Biol. 2001, $11,440-448$.

(48) Chen, S.; Tam, Y. C.; Lin, P.; Sung, M.; Tam, Y. K.; Cullis, P. R. Influence of Particle Size on the in Vivo Potency of Lipid Nanoparticle Formulations of SiRNA. J. Controlled Release 2016, 235, 236-244.

(49) Ramishetti, S.; Hazan-Halevy, I.; Palakuri, R.; Chatterjee, S.; Gonna, S. N.; Dammes, N.; Freilich, I.; Shmuel, L. K.; Danino, D.; Peer, D. A Combinatorial Library of Lipid Nanoparticles for RNA Delivery to Leukocytes. Adv. Mater. 2020, 32, 1906128.

(50) Kreiter, S.; Konrad, T.; Sester, M.; Huber, C.; Türeci, Ö.; Sahin, U. Simultaneous Ex Vivo Quantification of Antigen-Specific CD4+ and CD8+ T Cell Responses Using in Vitro Transcribed RNA. Cancer Immunol. Immunother. 2007, 56, 1577-1587.

(51) Blanchet, C. E.; Spilotros, A.; Schwemmer, F.; Graewert, M. A.; Kikhney, A.; Jeffries, C. M.; Franke, D.; Mark, D.; Zengerle, R.; Cipriani, F.; Fiedler, S.; Roessle, M.; Svergun, D. I. Versatile Sample Environments and Automation for Biological Solution X-Ray Scattering Experiments at the P12 Beamline (PETRA III, DESY). J. Appl. Crystallogr. 2015, 48, 431-443.

(52) Franke, D.; Kikhney, A. G.; Svergun, D. I. Automated Acquisition and Analysis of Small Angle X-Ray Scattering Data. Nucl. Instrum. Methods Phys. Res., Sect. A 2012, 689, 52-59.

(53) Ciccariello, S.; Goodisman, J.; Brumberger, H. On the Porod Law. J. Appl. Crystallogr. 1988, 21, 117-128.
(54) Seddon, J. M.; Cevc, G. Lipid Polymorphism:Lipid Polymorphism Structure and Stability of Lyotropic Mesophases of Phospholipids; 1993; DOI: $10.1201 / 9780203743577$. 